\title{
ORT.23 - Study to increase the disinfection holding time for classified areas at Bio-Manguinhos Formulation Division between two productive proces
}

Daniele Alves de Oliveira ${ }^{1 *}$; Marcos Vinícius de Souza Oliveira Antunes ${ }^{1}$; Ricardo Lacerda da Silva ${ }^{1}$; Felipe Leal da Silva ${ }^{1}$; Giselle Ribeiro de Barros ${ }^{1}$; Danielle Carneiro Vieira dos Santos ${ }^{1}$; Carla Cristina Vellasco do Nascimento Freixo ${ }^{1}$.

1Fiocruz/Bio-Manguinhos.

Introduction: The prevention of the existence and penetration of microorganisms in clean areas destined to the production of immunobiologicals and biopharmaceuticals is essential to meet the quality requirements of the products. Adequate disinfection of the facilities, equipment and utensils allows to meet these criteria. The area submitted to the sanitizing agents must maintain not only the cleaning conditions for a certain period without presenting a risk to the product to be processed, as well as allowing operational flexibility with safety. Increase the period of the sanitization between to the processes in the Clean Areas of the Formulation Division (DIFOR), in Bio-Manguinhos from 24 hours to 48 hours offered flexibility operational, reduction of exceptional activities with payment of overtime or increase of staff.

Objective: Increase the period of the sanitization between the productive processes in the clean areas of DIFOR to 48 hours, replacing the methodology currently used that considers the maximum interval of 24 hours between the cleanings.

Methodology: 1) Area monitored. Classified areas grade A and B, considering environmental monitoring points located near workstations and on critical surfaces such as the difficulty of cleaning and access, following a previous risk analysis and protocols in force. Three monitoring steps were carried out to prove the efficiency of study, as well as an aseptic validation race to incorporate the activity into the operational routine of DIFOR.

2) Desinfection and Environmental Monitoring. After the production process, sanitization was carried out only with sterile $70 \%$ alcohol solution, as it is the routine disinfectant, from the formulation rooms: CT-01, which is dedicated for measles, mumps, rubella (attenuated) vaccine and its estabilizers and CT-02 which is dedicated for the yellow fever vaccine (attenuated) and its antibiotics, contemplating the time and number of operators trained for the activity. The room remained without a productive process for 48 hours when sanitization was monitored, considering the environmental parameters: differences in pressure, temperature and relative humidity within specifications. The monitoring comprised plating samplings of contact surfaces (RODAC plates) and non-viable particles from the room, including the monitoring of viable particles (TSA/TSNI plates) of grade A area where the product is exposed during the process.

Results: The results obtained after reading the in CT-01 and in CT-02 monitored points were satisfactory, as well as the aseptic validations simulation of the filtration and distribution: stabilizers for the formulation of measles, mumps, rubella (attenuated) vaccine and antibiotics intended for the formulation of the yellow fever vaccine (attenuated).

Conclusion: The results showed the ability of the formulation room CT-01 and CT-02 to remain clean after a productive process for 48 hours, and permitted to introduce this practice at routine of production in to the clean areas of DIFOR, and can be extended for other areas in Bio-Manguinhos.

Keywords: Clean Area Disinfection; Environmental Monitoring; Aseptic Validation 